\title{
X-Ray Two-Photon Photoelectron Spectroscopy: A Theoretical Study of Inner-Shell Spectra of the Organic Para-Aminophenol Molecule
}

\author{
Robin Santra, ${ }^{1,2, *}$ Nikolai V. Kryzhevoi, ${ }^{3}$ and Lorenz S. Cederbaum ${ }^{3}$ \\ ${ }^{1}$ Argonne National Laboratory, Argonne, Illinois 60439, USA \\ ${ }^{2}$ Department of Physics, University of Chicago, Chicago, Illinois 60637, USA \\ ${ }^{3}$ Theoretical Chemistry, Institute of Physical Chemistry, Heidelberg University, Im Neuenheimer \\ Feld 229, 69120 Heidelberg, Germany
}

(Received 11 March 2009; published 1 July 2009)

\begin{abstract}
The inner-shell single and double ionization spectra of the organic molecule para-aminophenol are calculated using many-body Green's function methods. The inner-shell double ionization spectrum displays more pronounced sensitivity to the chemical environment and to electronic many-body effects than does the inner-shell single ionization spectrum. A kinetic model is employed to determine the probability of inner-shell double hole formation in para-aminophenol exposed to an intense, 1 fs $\mathrm{x}$-ray pulse. The resulting photoelectron spectrum at a photon energy of $1 \mathrm{keV}$ is calculated. This work suggests that x-ray two-photon photoelectron spectroscopy using x-ray free-electron lasers will provide access to electronic-structure information not currently available.
\end{abstract}

DOI: 10.1103/PhysRevLett.103.013002

PACS numbers: 33.60.+q, 31.15.A-, 33.80.Rv, 41.60.Cr

Photoelectron spectroscopy [1] is an outstanding technique for quantitatively characterizing the electronic structure of matter. Since the energy needed for the removal of an inner-shell electron is characteristic of the atomic species involved, inner-shell ionization using $\mathrm{x}$ rays allows one to determine the elemental composition of a sample. Furthermore, there is generally a measurable effect of the molecular environment on the binding energy of an innershell electron. This phenomenon is known as chemical shift. Traditional $\mathrm{x}$-ray photoelectron spectroscopy (XPS) is based on X-ray one-photon absorption. In this Letter, we demonstrate that the advent of $\mathrm{x}$-ray free-electron lasers [2-5] will enable a novel kind of photoelectron spectroscopy: x-ray two-photon photoelectron spectroscopy (XTPPS). XTPPS is more sensitive to chemical effects and electronic many-body effects than is traditional photoelectron spectroscopy.

Previous theoretical research on potential applications of $x$-ray free-electron lasers includes high-intensity $x$-ray absorption and scattering by atoms [6-9], nonlinear refraction of $\mathrm{x}$ rays in highly ionized condensed matter [10], $\mathrm{x}$-ray diffraction imaging of single biological molecules [11-14], and coherent x-ray nonlinear spectroscopy of molecules [15,16]. Similarly to Refs. $[15,16]$, the proposal presented in this Letter aims to exploit the high intensity of $\mathrm{x}$-ray free-electron laser pulses to reveal the electronic structure of molecules in a way not previously possible. However, in contrast to the concepts discussed in Refs. [15,16], XTPPS employs single x-ray pulses and does not require two independently tunable, mutually phase-coherent $\mathrm{x}$-ray pulses.

The basic idea underlying XTPPS is the following. Assume that the $\mathrm{x}$-ray photon energy is chosen to be much higher than any inner-shell threshold of interest in a given molecule. Assume further that during a given $\mathrm{x}$-ray pulse the molecule absorbs an x-ray photon and an innershell hole is formed. The associated photoelectron has a kinetic energy that is characteristic of the site within the molecule where the hole was formed. If the intensity of the $\mathrm{x}$-ray pulse is high enough, a second $\mathrm{x}$-ray photon may be absorbed before the $\mathrm{x}$-ray pulse is over. If, in addition, the $\mathrm{x}$-ray pulse is short in comparison to the decay lifetime of the inner-shell vacancy, then it is virtually guaranteed that the second x-ray photon is absorbed by the inner-shellexcited state prepared by the first $\mathrm{x}$-ray photon absorbed by the molecule. The kinetic energy of the second photoelectron ejected reflects the electronic structure of the innershell-excited molecular ion. By monitoring the entire photoelectron spectrum as a function of the peak intensity of the x-ray pulse, one can identify conditions under which no more than two x-ray photons are absorbed during the $\mathrm{x}$-ray pulse.

The inner-shell double ionization spectra that may be measured using XTPPS can be connected to electronic many-body theory using Green's functions or propagators [17]. For XPS, the appropriate Green's function is the oneparticle propagator [18]. The pole positions of the oneparticle propagator yield the molecular ionization potentials, i.e., the energy of the cationic eigenstates relative to the energy of the neutral ground state. The residuum or pole strength of a pole of the one-particle propagator is a measure of how well a cationic eigenstate is described within the one-hole configuration space deriving from the Hartree-Fock ground state of the neutral molecule. In other words, the pole strength is a measure of the independentparticle character of a specific ionized state. The Green's function appropriate for XTPPS is the particle-particle propagator [19]. The pole positions of the particle-particle 
propagator yield the double ionization potentials, i.e., the energy of the dicationic eigenstates relative to the energy of the neutral ground state. The pole strength of a pole of the particle-particle propagator is a measure of how well a dicationic eigenstate is described within the two-hole configuration space.

Inner-shell double vacancies are more sensitive to the chemical environment of the inner-shell-excited atoms than are inner-shell single vacancies [20,21]. The chemical shifts in inner-shell double ionization spectra are particularly pronounced when each of the two inner-shell holes is at a different atomic site. Many-body effects in double core ionization spectra can be so strong that the molecularorbital picture of double ionization breaks down [22]. In order to demonstrate the feasibility of XTPPS using x-ray free-electron lasers, we present in this Letter calculations on the organic molecule para-aminophenol.

Figure 1 displays the inner-shell single and double ionization potentials of para-aminophenol. The propagators involved were evaluated using a sophisticated perturbation-theoretical approximation known as the algebraic diagrammatic construction (ADC) scheme [19,23]. The $n$ th-order $\mathrm{ADC}$ scheme, $\mathrm{ADC}(n)$, is complete through $n$th order and includes in addition infinite summations over certain classes of Feynman diagrams. Within ADC, the pole positions and pole strengths of a Green's function are found by solving a symmetric eigenvalue problem.

The inner-shell single ionization spectrum in Fig. 1(a) was calculated using the ADC(4) method [23,24]. In the ADC(4) method implemented, the configuration space comprises the inner-shell one-hole configurations and all their single and double excitations. We used $\operatorname{ADC}(2)$ $[19,25]$ to calculate the double ionization spectrum in Fig. 1(b). The configuration space in this method comprises all two-hole configurations and all their single excitations. The ADC methods require Hartree-Fock orbitals and their energies as input data. The Hartree-Fock calculation was performed using the $6-31 \mathrm{G}$ basis set for the

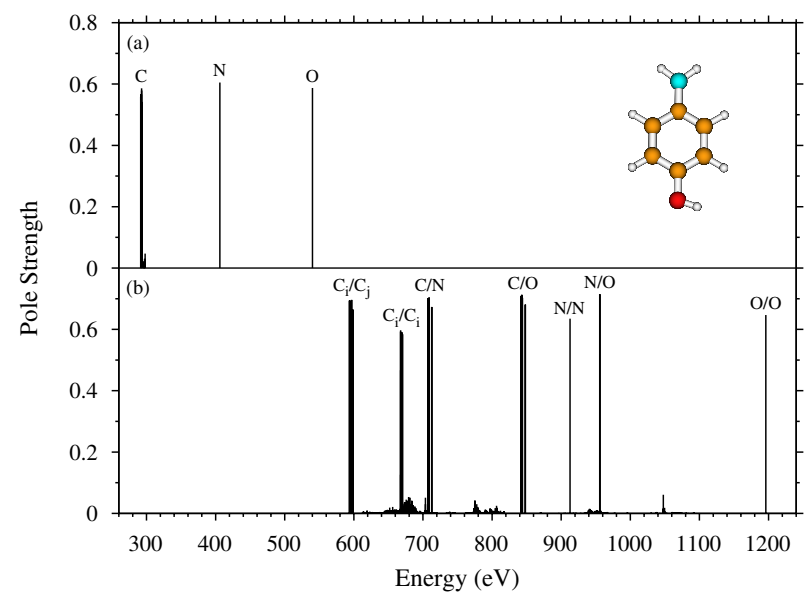

FIG. 1 (color online). Inner-shell single (a) and double (b) ionization potentials of para-aminophenol calculated using many-body Green's function methods.
$\mathrm{C}$ and $\mathrm{H}$ atoms. The $6-31 \mathrm{G}^{*}$ basis set [26] was placed on the $\mathrm{N}$ and $\mathrm{O}$ atoms.

There are three distinct groups of lines in the inner-shell single ionization spectrum of para-aminophenol [Fig. 1(a)]. These groups correspond to $K$-shell ionization of the atomic species C, N, and O, respectively. Since there are six distinguishable $\mathrm{C}$ atoms in para-aminophenol, the $\mathrm{C}$ $1 s$ main lines are split up by chemical shifts. The effect, however, is rather small. The weak satellites right above the $\mathrm{C} 1 s$ main lines are a manifestation of electronic manybody effects associated with the removal of a $\mathrm{C} 1 \mathrm{~s}$ electron.

By contrast, the inner-shell double ionization spectrum of para-aminophenol [Fig. 1(b)] is much richer. In this case, there are seven groups of lines. For instance, a $1 \mathrm{~s}$ vacancy on one $\mathrm{C}$ atom may be accompanied by a $1 s$ vacancy on another $\mathrm{C}$ atom. This is symbolized by $\mathrm{C}_{i} / \mathrm{C}_{j}$ in Fig. 1(b); $C_{i} / C_{i}$ indicates that both $1 s$ holes reside on the same $\mathrm{C}$ atom. It is apparent that the chemical shifts for all groups characterized by at least one $\mathrm{C} 1 s$ hole are more pronounced than for the $\mathrm{C} 1 s$ single ionization lines in Fig. 1(a). The large number of satellites surrounding the $\mathrm{C}_{i} / \mathrm{C}_{i}$ lines highlights the importance of many-body effects in inner-shell double ionization spectra. Owing to the fact that the particle-particle propagator of an interacting many-electron system cannot be expressed solely in terms of the properties of the one-particle propagator, the innershell double ionization spectrum in Fig. 1(b) contains fundamentally new information not available from the innershell single ionization spectrum in Fig. 1(a). This includes information on the interaction between two core holes and on the electronic structure between the two holes.

It is possible, in principle, to create inner-shell double vacancies via one-photon absorption [27,28]. For molecules, however, this approach has disadvantages. First, double inner-shell ionization via one-photon absorption has a relatively small probability (typically less than a percent of the single ionization probability). Second, when two inner-shell electrons are ejected via one-photon absorption, they generally come from a single atomic site. Therefore, the two-site $\mathrm{C}_{i} / \mathrm{C}_{j}$ lines [Fig. 1(b)], for example, are not accessible.

XTPPS employing x-ray free-electron lasers will overcome these limitations. It is expected that using the technique described in Ref. [29] it will be possible at the Linac Coherent Light Source [3] to generate 1.2 fs pulses containing up to $2.4 \times 10^{11}$ photons at an $\mathrm{x}$-ray photon energy in the vicinity of $1 \mathrm{keV}$ [30]. Such X-ray pulses are much shorter than the Auger lifetime of either $\mathrm{C}, \mathrm{N}$, or $\mathrm{O} 1 \mathrm{~s}$ vacancies. (The only slightly critical case is an O $1 s$ hole, which has an Auger lifetime of 5 fs [31].) Auger decay during the $\mathrm{x}$-ray pulse is therefore unlikely and is neglected in the following.

We now show that such x-ray pulses, when focused to a width of $1 \mu \mathrm{m}$, are intense enough to enable XTPPS. Assuming a photon energy of $1 \mathrm{keV}$, we are in the extended $\mathrm{x}$-ray absorption fine-structure regime for both single and 
double hole formation. For instance, it follows from Fig. 1 that the $\mathrm{O} 1 s$ electron binding energy is $540 \mathrm{eV}$; when an $\mathrm{O} 1 s$ hole is present, the binding energy for the remaining $\mathrm{O} 1 s$ electron is $1200-540=660 \mathrm{eV}$. The photoelectron energy is therefore at least $340 \mathrm{eV}$. To a first approximation, the molecular x-ray absorption cross section so high above the respective edges is given by the sum of the $\mathrm{x}$-ray absorption cross sections of the atomic constituents of the molecule. We determined the photoionization cross sections for the various inner-shell single and double hole atomic channels by numerically integrating the radial Schrödinger equation for the corresponding HartreeSlater atomic potential [32].

The atomic photoionization cross sections serve as input parameters for a kinetic model of molecular inner-shell single and double ionization. Within this model, we solve 14 coupled rate equations for a given $\mathrm{x}$-ray pulse. Each equation describes the time evolution of one of the 14 channels that we consider. One channel, for example, is the ground state of para-aminophenol. Before the x-ray pulse, the population in this channel is unity. Another channel is the manifold of states in which one of the six $\mathrm{C}$ atoms in para-aminophenol has a $1 s$ hole. (There is no second hole present in this channel.) For the double hole channels associated, for example, with a $\mathrm{C} / \mathrm{O}$ double hole state (cf. Fig. 1), it is important for the determination of the photoelectron spectrum to distinguish between the possible excitation pathways, i.e., whether $\mathrm{C}$ is ionized first and $\mathrm{O}$ second, or vice versa. We neglect any channels involving one or more valence holes. The associated cross sections are relatively small.

Figure 2 shows the channel populations obtained with the kinetic model. Early in the x-ray pulse, single ionization via one-photon absorption takes place. Even though the $\mathrm{O} 1 s$ and $\mathrm{N} 1 s$ photoionization cross sections are larger than the atomic $\mathrm{C} 1 s$ photoionization cross section, the C $1 s$ hole population dominates because in paraaminophenol there are 6 times more $\mathrm{C}$ atoms than $\mathrm{N}$ or $\mathrm{O}$ atoms. The single hole populations reach their maximum before the peak of the x-ray pulse. The absorption of a second photon transfers the population from the single hole channels to the double hole channels. Two-photon double ionization saturates after the peak of the x-ray pulse. Here, we focus on the two-photon case and neglect averaging effects due to the spatial profile of the x-ray pulse. In practice, it is necessary to measure the photoelectron spectrum as a function of the $\mathrm{x}$-ray peak intensity in order to monitor whether additional $\mathrm{x}$-ray photons are absorbed before the $\mathrm{x}$-ray pulse is over. By introducing suitable many-body Green's functions, the ideas presented in this Letter can be extended to three-photon or higher-order processes.

Using the Green's function spectra from Fig. 1 and the channel populations at the end of the x-ray pulse (Fig. 2), we constructed the photoelectron spectrum plotted in Fig. 3. Satellite lines are not shown in the figure. To give a specific example of how the photoelectron spectrum was

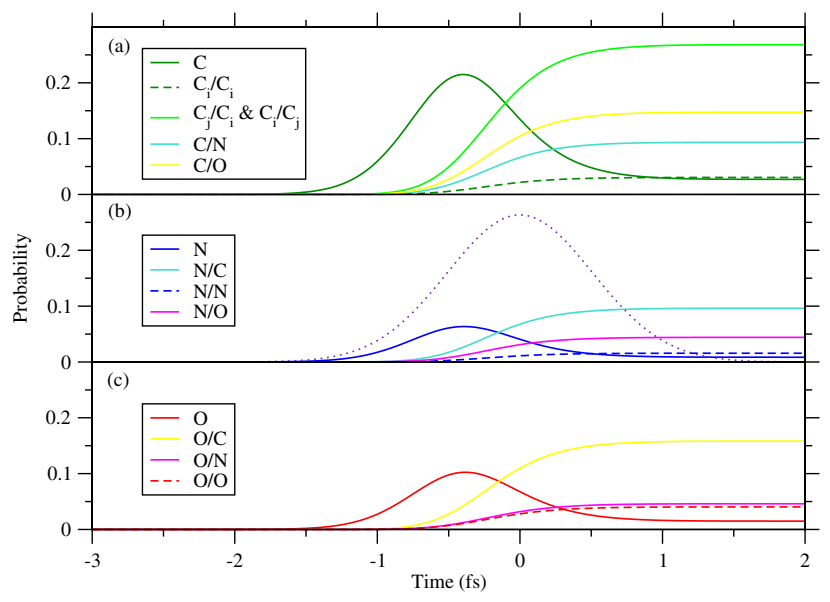

FIG. 2 (color online). Time evolution of the inner-shell single and double ionization channels of para-aminophenol exposed to a 1.2-fs, 1-keV x-ray pulse. The pulse is assumed to contain $1.0 \times 10^{11}$ photons focused to a width of $1 \mu \mathrm{m}$. (a) In the channels shown, the first photon absorbed creates a $\mathrm{C} 1 s$ hole. (b) The first photon absorbed creates an $\mathrm{N} 1 s$ hole. (c) The first photon absorbed creates an $\mathrm{O} 1 s$ hole. The dotted curve in (b) indicates the Gaussian temporal profile of the X-ray pulse.

determined, consider the case where a $\mathrm{C} 1 s$ electron is ejected first. The corresponding channels are shown in Fig. 2(a). We took the group of $\mathrm{C} 1 s$ main lines from the single ionization spectrum in Fig. 1(a) and placed them in the photoelectron spectrum by subtracting the respective binding energies from the photon energy of $1 \mathrm{keV}$. The intensities of the $\mathrm{C} 1 s$ photoelectron lines were renormalized so that their sum equals the sum of the channel populations in Fig. 2(a).

Consider next the second photoelectron associated, for example, with the $\mathrm{C}_{j} / \mathrm{C}_{i}$ and $\mathrm{C}_{i} / \mathrm{C}_{j}$ two-site channels. This notation indicates that there are two distinct lines for the energy of the second photoelectron for a given two-site $\mathrm{C}_{i} / \mathrm{C}_{j}$ main line in the double ionization spectrum in

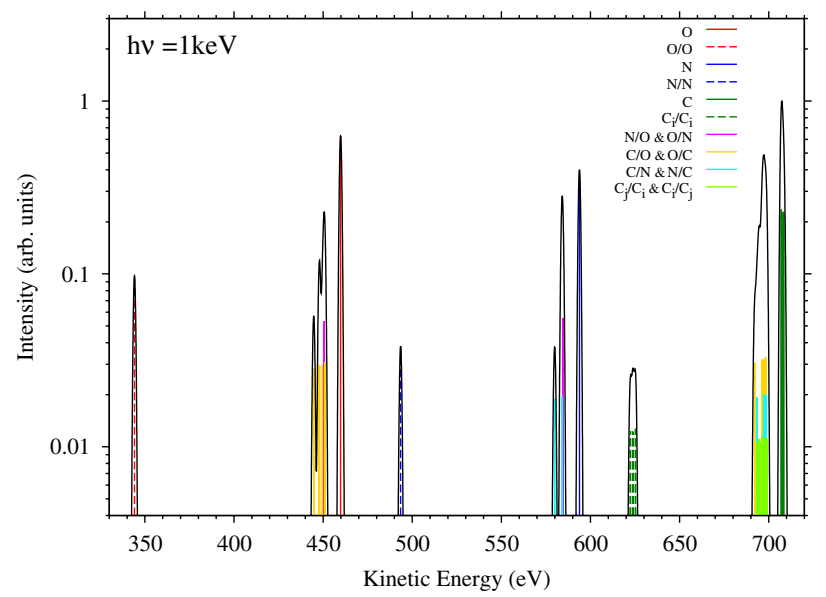

FIG. 3 (color online). Photoelectron spectrum of paraaminophenol (photon energy $1 \mathrm{keV}$, pulse duration $1.2 \mathrm{fs}$, number of photons $1.0 \times 10^{11}$, focal width $\left.1 \mu \mathrm{m}\right)$. 
Fig. 1(b). We determined the electronic character of $\mathrm{C}_{i}$ and $\mathrm{C}_{j}$, respectively, and identified from Fig. 1(a) the corresponding single ionization energies $I_{i}^{(1)}$ and $I_{j}^{(1)}$. Let $I_{i, j}^{(2)}$ denote the double ionization potential for the $\mathrm{C}_{i} / \mathrm{C}_{j}$ main line under consideration. One line for the second photoelectron then appears at an electron energy of $1 \mathrm{keV}$ $-\left[I_{i, j}^{(2)}-I_{i}^{(1)}\right]\left(\mathrm{C}_{i} / \mathrm{C}_{j}\right.$ channel $)$; the second line appears at $1 \mathrm{keV}-\left[I_{i, j}^{(2)}-I_{j}^{(1)}\right]\left(\mathrm{C}_{j} / \mathrm{C}_{i}\right.$ channel $)$. After taking into account the spin multiplicity of the double hole states, the sum of the photoelectron line intensities for the $\mathrm{C}_{j} / \mathrm{C}_{i}$ and $\mathrm{C}_{i} / \mathrm{C}_{j}$ channels was renormalized to the $\mathrm{C}_{j} / \mathrm{C}_{i}$ and $\mathrm{C}_{i} / \mathrm{C}_{j}$ population in Fig. 2(a). Using the same logic, all other photoelectron lines were constructed.

Because we assume here an $\mathrm{x}$-ray pulse duration of $1.2 \mathrm{fs}$, the spectral bandwidth of the x-ray pulse is $1.5 \mathrm{eV}$. Therefore, in addition to the discrete photoelectron lines associated with individual electronic states, we show in Fig. 3 the photoelectron spectrum obtained by convolving the discrete photoelectron lines with a Gaussian of width $1.5 \mathrm{eV}$ (full width at half maximum). Other broadening mechanisms, including the instrumental resolution, are less important.

The x-ray two-photon-induced photoelectron spectrum of para-aminophenol (Fig. 3) may be divided into three disjunct energy regions. The region between 340 and $460 \mathrm{eV}$ corresponds to electronic states with at least one $\mathrm{O} 1 s$ hole. Photoelectrons in the region between 490 and $600 \mathrm{eV}$ signal the formation of at least one $\mathrm{N} 1 s$ hole. At least one $\mathrm{C} 1 s$ hole is present in the electronic states giving rise to the photoelectrons in the region between 620 and $710 \mathrm{eV}$. In each region, the peak associated with the highest kinetic energy corresponds to a photoelectron ejected by the absorption of the first photon in the sequential twophoton process considered here. This photoelectron line is also present in spectra measured at low x-ray intensity. The peak appearing at the lowest kinetic energy in a given region corresponds to the second photoelectron ejected during the formation of a one-site double hole state. The remaining peaks indicate two-site double hole states.

The inner-shell-excited states relax via Auger decay. The kinetic energy spectrum of the Auger electrons overlaps with the photoelectron spectrum in Fig. 3. As in traditional one-photon photoelectron spectroscopy, this causes no difficulty. One can always distinguish the Auger electrons from the photoelectrons by changing the photon energy. The computational study performed in this Letter suggests that XTPPS using $\mathrm{x}$-ray free-electron lasers is likely to become a powerful tool for probing the innershell particle-particle propagator of molecules. The innershell double ionization spectra accessible via XTPPS are much richer, and chemically more sensitive, than are traditional inner-shell single ionization spectra.

R. S. thanks Nora Berrah, Ryan Coffee, John Galayda, Elliot Kanter, and Linda Young for inspiring discussions. R.S. was supported by the Office of Basic Energy
Sciences, Office of Science, U.S. Department of Energy, under Contract No. DE-AC02-06CH11357. N. K. and L. C. gratefully acknowledge financial support by the DFG.

*Corresponding author.

[1] S. Hüfner, Photoelectron Spectroscopy (Springer, Berlin, 1996).

[2] J. Feldhaus, J. Arthur, and J. B. Hastings, J. Phys. B 38, S799 (2005).

[3] J. Arthur et al., Linac Coherent Light Source (LCLS) Conceptual Design Report No. SLAC-R-593, 2002, http://www-ssrl.slac.stanford.edu/lcls/cdr/

[4] Riken SCSS X-FEL Conceptual Design Report, edited by T. Tanaka and T. Shintake, 2005, http://www-xfel. spring8.or.jp/.

[5] DESY Technical Design Report No. 2006-XXX, edited by M. Altarelli et al., 2006, http://xfel.desy.de/tdr/index_ eng.html

[6] N. Rohringer and R. Santra, Phys. Rev. A 76, 033416 (2007).

[7] S. P. Hau-Riege, Phys. Rev. A 76, 042511 (2007).

[8] K. Moribayashi, Phys. Rev. A 76, 042705 (2007).

[9] N. Rohringer and R. Santra, Phys. Rev. A 77, 053404 (2008).

[10] C. Conti et al., Opt. Express 16, 8324 (2008).

[11] R. Neutze et al., Nature (London) 406, 752 (2000).

[12] S. P. Hau-Riege et al., Phys. Rev. Lett. 98, 198302 (2007).

[13] K. E. Schmidt et al., Phys. Rev. Lett. 101, 115507 (2008).

[14] R. Fung et al., Nature Phys. 5, 64 (2009).

[15] S. Tanaka and S. Mukamel, Phys. Rev. Lett. 89, 043001 (2002).

[16] I. V. Schweigert and S. Mukamel, Phys. Rev. Lett. 99, 163001 (2007).

[17] A. L. Fetter and J. D. Walecka, Quantum Theory of ManyParticle Systems (McGraw-Hill, Boston, 1971).

[18] L. S. Cederbaum and W. Domcke, Adv. Chem. Phys. 36, 205 (1977).

[19] J. Schirmer and A. Barth, Z. Phys. A 317, 267 (1984).

[20] L. S. Cederbaum et al., J. Chem. Phys. 85, 6513 (1986).

[21] L. S. Cederbaum et al., J. Chem. Phys. 86, 2168 (1987).

[22] E. M.-L. Ohrendorf, L. S. Cederbaum, and F. Tarantelli, Phys. Rev. A 44, 205 (1991).

[23] J. Schirmer, L. S. Cederbaum, and O. Walter, Phys. Rev. A 28, 1237 (1983).

[24] G. Angonoa, O. Walter, and J. Schirmer, J. Chem. Phys. 87, 6789 (1987).

[25] F. O. Gottfried, L. S. Cederbaum, and F. Tarantelli, Phys. Rev. A 53, 2118 (1996).

[26] P. C. Hariharan and J. A. Pople, Theor. Chim. Acta 28, 213 (1973).

[27] S. H. Southworth et al., Phys. Rev. A 67, 062712 (2003).

[28] J. Hoszowska et al., Phys. Rev. Lett. 102, 073006 (2009).

[29] Y. Ding et al., Phys. Rev. Lett. 102, 254801 (2009).

[30] J. Galayda (private communication).

[31] D. L. Walters and C.P. Bhalla, Phys. Rev. A 3, 1919 (1971).

[32] S. T. Manson and J.W. Cooper, Phys. Rev. 165, 126 (1968). 